\title{
Purpose and Design of a Digital Environment for the Professional Insertion of Students Based on the E-portfolio Approach
}

\author{
https://doi.org/10.3991/ijet.v17i01.26703 \\ Hanae Mgarbi ${ }^{(\bowtie)}$, Mohamed Yassin Chkouri, Abderrahim Tahiri \\ National School of Applied Science Tetouan, Abdelmalek Essaadi University (UAE), Tetouan, \\ Morocco \\ hanae.mgarbi@etu.uae.ac.ma
}

\begin{abstract}
Before the development of the web, we talk about the portfolio; it was represented by a simple portfolio of student skills in paper form. This form of representation of skills and professional career has continued to develop over the years. After a succession of several stages in the concept of the portfolio, this simple paper form was transformed into a digital form called an e-portfolio [1] [5], which is centered on the personal and collective learning dynamics throughout the life of the student [10] [22]. In the digital age, the curriculum vitae (CV) is the first door to professional integration [7]. But the paper CV remains a document too linear and has not adapted to the complexity of current modes and channels of communication. The objective of our research work is the purpose and design of a digital environment for the professional insertion of students based on the e-portfolio, allowing students to build and enhance academic and extra-academic achievements while being part of a lifelong learning approach, to develop their digital visibility by capitalizing on training achievements and skills obtained, and to present themselves digitally.
\end{abstract}

Keywords-e-portfolio, lifelong learning, digital identity, CV, digital environment

\section{Introduction}

Faced with companies wishing to find suitable and operational profiles, graduates leave university with acquired knowledge and skills which they group together and present in the form of curriculum vitae (CV) which is the first door to professional integration. But at the present time, the paper $\mathrm{CV}$ remains a document too linear when in reality the paths are more complex and more multiple. This form of representation of skills and professional career has not ceased to develop throughout these years, since this form does not facilitate for companies the search for trainees and suitable employees according to their sector of activity and professional needs. In addition, this document presenting the student profile has not adapted to the complexity of current modes 
and channels of communication. University laureates must be able to present themselves digitally. Currently, the student must be able to think about his professional integration in a bundle of constructions, proofs and demonstrations of skills.

In fact, the e-portfolio approaches [1] [5] [9] is part of a movement at the heart of digital transformations. It is about empowering and encouraging the student to build his life on his own, to build himself and to construct his itinerary in order to present it better [10]. This approach is centered on the dynamics of personal and collective learning throughout the student's life [22] and on the development of the culture of digital identity by the student by the valuation of a reflective approach on their personal project: personal, professional and training course. This form allows at the same time, for universities and for students, to archive and show their learning processes, to promote their knowledge, and thus to give students more chance to create a professional network with companies.

Several e-portfolios have been emerged with different characteristics [2] [3] [6]. A study on open-source platforms was carried out based on several criteria including ergonomics, organization, security, sharing, and communication. Four platforms (Mahara [11], Karuta [14], Canvas [16] and Exabis [17]) were selected for this study to identify the advantage and inconvenient for each platform, and to identify the most important element to compose an e-portfolio.

The main objective of our research work is establishment of a digital environment based on the e-portfolio approach allowing students to develop their digital visibility by capitalizing on the learning outcomes and skills obtained during their studies and after graduation. This work was achieved by defining the set of features based on the e-portfolio approach and distributed to the sections defined to construct the student eportfolio. The architecture and the implementation of the platform are based on the novel technologies, deployed and experimented on by fixed group of the students to be evaluated. The purpose of the platform is to be a tool oriented towards assisting the professional integration of graduates into the socio-economic world. Our approach has several advantages as the promotion of a reflective approach [7] by students on their personal project: personal, professional and training course (initial, continuing and throughout life) and the development of the culture of digital identity by the student.

This paper is organized as follows. Section 2 gives an overview of e-portfolio approach. We discuss the related works at the Section 3. Section 4 presents the functionalities, architecture and platform based on e-portfolio approach. Conclusions close the article in Section 5.

\section{The e-portfolio approach}

If the portfolios [1] [5] have been used in the field of education in paper format in order to present the work of the students and thus demonstrate their development, progress and achievements. With the evolution of the Internet, «classic» portfolios have taken the form of «electronic portfolios» or «e-portfolios»; they have been used in educational [4] and professional fields. The electronic version of the portfolio [9] [21] has additional potential and possibilities: flexibility, ease of modification of the contents 
and structure of the portfolio, the addition of multimedia documents, accessibility and integration into a large network.

The e-portfolio [19] [20] can be defined as «an evolving set of documents and electronic resources capitalized in a digital environment describing and illustrating the learning, experience, skills or background of its author. Accessible remotely via interoperable technology, it is based on a personal database (information, documents or links accessible via the Internet) and one (or more) collective selective publication space (s).» («the e-portfolio approach in higher education» [19])

There are four types of e-portfolio approach: presentation, learning, personal development and evaluation. In this article, the research work is focused on the development of a presentation e-portfolio.

\subsection{Characteristics of the e-portfolio}

An e-portfolio is based on archiving and promoting the skills and training acquired throughout the student's life [22]. Indeed, the author of the e-portfolio must be able to master the content and services of this tool in order to:

- Capitalize on their experiences while providing proof of mastery of skills (scientific, engineering, social, etc.).

- Support their professional integration and personal development throughout life.

- Stand out by the singularity of their experiences, their project and their career.

- Strengthen its reputation on the internet; as well as the notoriety of its establishment.

- Promote guidance, mobility and training throughout one's professional life.

- Create a network with other students and laureates, in order to promote internship and professional integration, and then have knowledge with other recruiters and employers.

\subsection{Purpose of the e-portfolio}

The e-portfolio, as an approach and a tool, can be seen as a possible response to some of today's demands from an educational perspective. On the one hand, education is no longer seen as only a basis for professional life, but also as a continuous lifelong [22] commitment, driven by expectations in terms of the development of knowledge and skills. On the other hand, generalized access to information and technology today opens up great flexibility and internationalization of education.

Some of these changes concern the professional integration of students and future researchers: The competitiveness of our globalized society requires people to have a higher level of self-awareness. Therefore, the development of metacognitive skills learning to acquire skills - is crucial. The ability to show the high quality of training received and the learning outcomes achieved makes e-portfolios interesting for students as well as for institutions; they provide transparency and a better understanding of student skills. 


\subsection{Principles of the e-portfolio}

The e-portfolio approach is based on the idea of guaranteeing a dedicated space for individuals to collect, deposit, discuss, share and arrange everything that concerns them. It is based on the following principles:

Develop a profile and a digital identity. The student should think of his e-portfolio as a database about himself and his experience [8]. He must therefore include not only the elements making it possible to identify him, but also his work, his studies, his certifications, his colleagues' testimonies and letters of recommendation. The goal is therefore to present and to promote yourself.

Have a clear idea of their strengths and weaknesses. Each registered student must be able to present their skills acquired throughout their life and then qualify in relation to each skill, while assigning a percentage expressing their level in relation to this skill, which allows you to have a clear idea of your strengths and weaknesses [12].

The content of the e-portfolio belongs to the student and is a matter of private life. It is he who chooses which data will be visible (public) and which will be invisible. A guarantee of reading rights must allow the student to have full visibility of his digital identity and therefore of the data that can be published.

\section{$3 \quad$ Related works}

In this section, we conducted a study on open-source platforms based on several criteria including ergonomics, organization, security, sharing, and communication. Four platforms (Mahara, Karuta, Canvas and Exabis) were selected for this study.

- Mahara': Focused on an e-portfolio for the enhancement of skills and adapted to the creation of content in a fairly free manner by the student. Mahara [11] [18] aims for a type of e-portfolio that is very free in terms of creation by the student and very suitable for the implementation of the e-portfolio for valuing skills, achievements and presentation. This solution offers a lot of flexibility for the author's free creation of collections and pages, writings, information arrangements, document integrations that he can share related to skills. Its other great strength is the possible integration with the LMS Moodle [13] widely used in university training. It can also be used as a daily logbook in which the student describes and reflects quite freely on his learning [3]. These two strengths make it a particularly effective tool from a student's point of view for documenting and sharing learning outcomes.

- Karuta ${ }^{2}$ : is a flexible tool and development environment that allows you to create all types of e-portfolios [14]. It is possible to create sections, pages and subsections to organize the information architecture [3]. Its biggest strength lays in the infinite possibilities of combinations and communication between objects that can be created by the designer of the e-portfolio such as, for example, made-to-measure repositories with evaluation criteria, which can be called up by the user, by personalized menus, or selected by lists of categories of repositories, or integrated directly into the pages.

\footnotetext{
${ }^{1}$ https://mahara.org/

${ }^{2}$ https://karuta-france-portfolio.fr/
} 
Editing pages allows less flexibility than in Mahara: no dragging and dropping of content, less free formatting of pages by the author. Karuta does not integrate with an LMS but is LTI compatible, which allows you to develop interactions with Moodle, Sakai, etc.

- Canvas ${ }^{3}$ : Canvas LMS [16] is an LMS published by the company Instructure, it is open source and free. The "standard" version targets higher education institutions [4], and is used extensively by American universities. The K-12 version is intended for primary and secondary schools. There is also Canvas Network [15], which offers free MOOCs (Massive Open Online Course) on various subjects (science, history, ecology...). Finally, Canvas Bridge is the corporate version of the LMS. Canvas LMS really sets itself apart with its easy-to-use and intuitive graphical interface. The ergonomics are really good, which is not necessarily the case with a lot of other LMS. It natively integrates an e-portfolio interface with all the basic functionalities but with quite limited options.

- Exabis ${ }^{4}$ : Exabis ePortfolio allows students to create, collect and share outcomes of their learning process in form of a digital portfolio. This block can be easily added to a Moodle installation and provides e-portfolio functionality for student and teacher. Students can build their e-portfolios by collecting web links, files and notes in an individually defined category system. Artifacts can be assembled into views that can be published for specific target groups, they receive thoughts and comments submitted by others, as well as tasks from the Moodle installations [17]. In addition, the eportfolio can be saved as a SCORM package, which is a standardized schema and file format for the transport of training data and it can also be used on multiple platforms for further work.

\section{Digital environment based on the e-portfolio approach}

In this section, we will first present the functionalities of the e-portfolio platform following our studies on e-portfolio approach. Then we will describe the sequence diagram for student registration and the software architecture of the e-portfolio platform. Finally, we will present the online e-portfolio platform.

\subsection{E-portfolio platform functionalities}

The e-portfolio platform contains a protected area, i.e. any account added must be protected by an identification consisting of a login (its e-portfolio number) and a password. For the creation of a student user account two steps are necessary: The first step, the student must complete a registration form indicating his student number, a valid email address, the home university and a password. The second step, a validation email is sent to the student to allow him to continue with the registration process. Email validation is very important for creating any account. Following the registration step and

\footnotetext{
${ }^{3}$ https://www.instructure.com/en-gb/canvas

${ }^{4}$ https://moodle.org/plugins/block_exaport
} 
the creation of his login details, the user will be automatically directed to a dedicated protected area allowing him to enter personal information about him.

Once the user is authenticated, he will have the right to access the settings area which allows him to modify and complete all his personal data and choose the navigation language. The user will have the choice between the French language and the Arabic language. Then, the user is automatically directed to the main page of the platform, where he will have the right to access all of the following features:

The student can complete part or all of his e-portfolio through the 10 sections offered:

1. Who am I?

2. My presentation video

3. My training

4. My professional experiences

5. My skills

6. My projects

7. My recommendations and certifications

8. My languages

9. My extra-curricular and leisure activities

10. Contact me

Modification of the visibility of data and personal and professional information entered. This means that the student will have the possibility to publish (and therefore to make visible) part or all of his data. Changing the visibility of the data can be done with a single click according to the 10 section described above.

Visualization of the CV in web or in PDF formats. The student will be able to share his CV with companies in both PDF and web formats. The web CV contains the video and links of the student's supporting documents while the PDF CV contains only textual information.

Other features will be expected at the level of the e-portfolio platform, such as:

1. Access to a selective publications area (internship and job offers): Depending on the interests of the user-student declared during registration, publications will be displayed on their home page.

2. Access to spaces and profiles of companies registered on the platform to consult their internship or job offers. If the student is interested in an offer, he can apply automatically and directly to said company.

3. Search for companies and their internship / job offers, by entering the field of application of a company, the sector of activity, and the region, etc.

\subsection{Registration sequence diagram}

Sequence diagrams are the graphic representation of interactions between actors and the system in chronological order. They make it possible to describe how the elements of the system interact with each other and with the actors. In this section we will show a single sequence diagram for student registration as shown in Figure 1. 


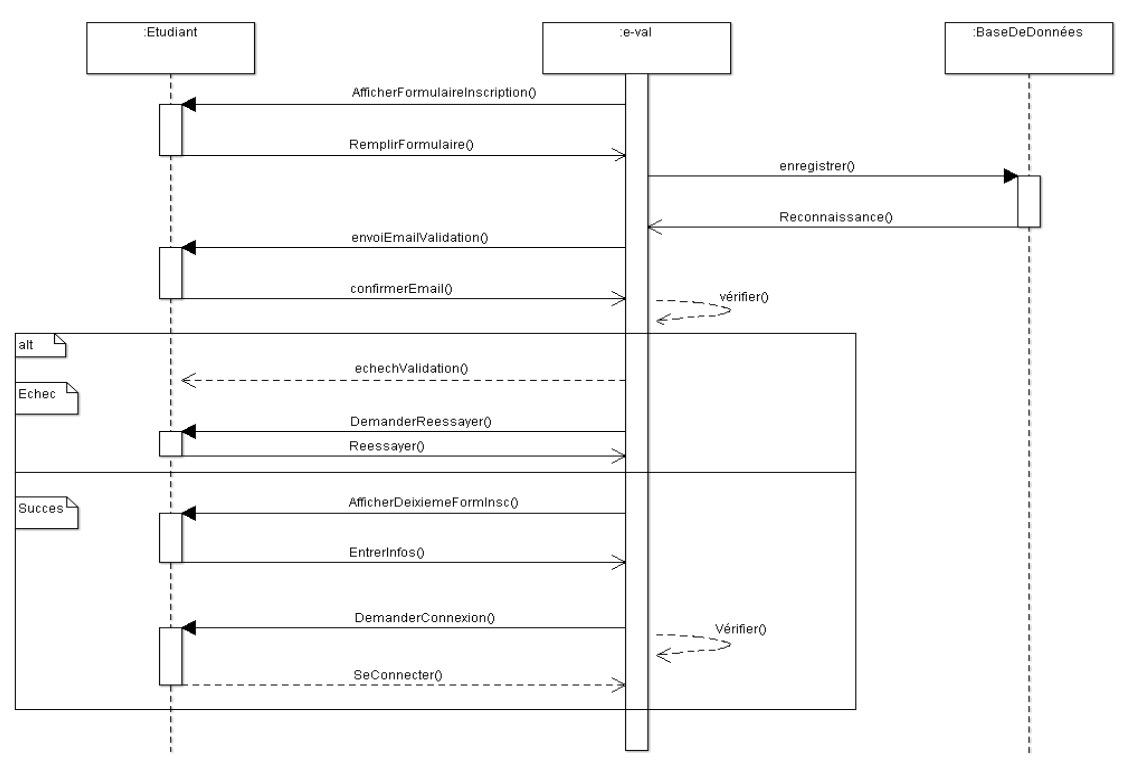

Fig. 1. Sequence diagram for student registration

For a student, the registration is done in two steps. The first step is to verify and save the information in the database, and the second is to send a validation email:

- If the validation was successful, a new registration window will be displayed by the platform.

- If there is a validation failure, the student will be invited to repeat their request until they can validate their email and thus continue their registration on the platform.

\subsection{Software architecture of the platform}

The platform architecture is presented in the form of three parts as shown in Figure 2. The first represents the user view, the second represents the back end (processes), and the third represents the noSQL MongoDB database.

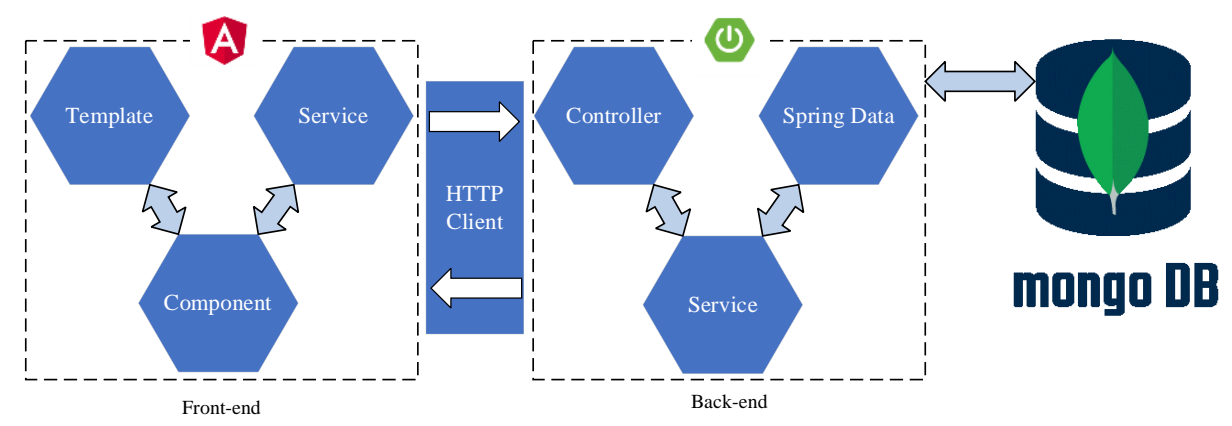

Fig. 2. Software architecture of the e-portfolio platform 
The front-end part is composed by the Components that allow you to view data on the screen, listen to user-generated events, and initiate actions; by the Template represented by the HTML / SCSS pages; and the Service that allows data to be submitted through a POST request sent to the REST API.

The back end portion of the e-portfolio platform is composed by the Controller layer that contains all of the application's service code; the Service layer that contains all the entities; and the $D A O$ layer allowing access to the database through the Spring Data Framework.

\subsection{Online e-portfolio platform}

The e-portfolio platform has been deployed and experimented by Moroccan universities on the following link: eval.uae.ac.ma. Figure 3 shows the main part of the e-portfolio, from which the student can fill in all the elements mentioned in section 2.1.

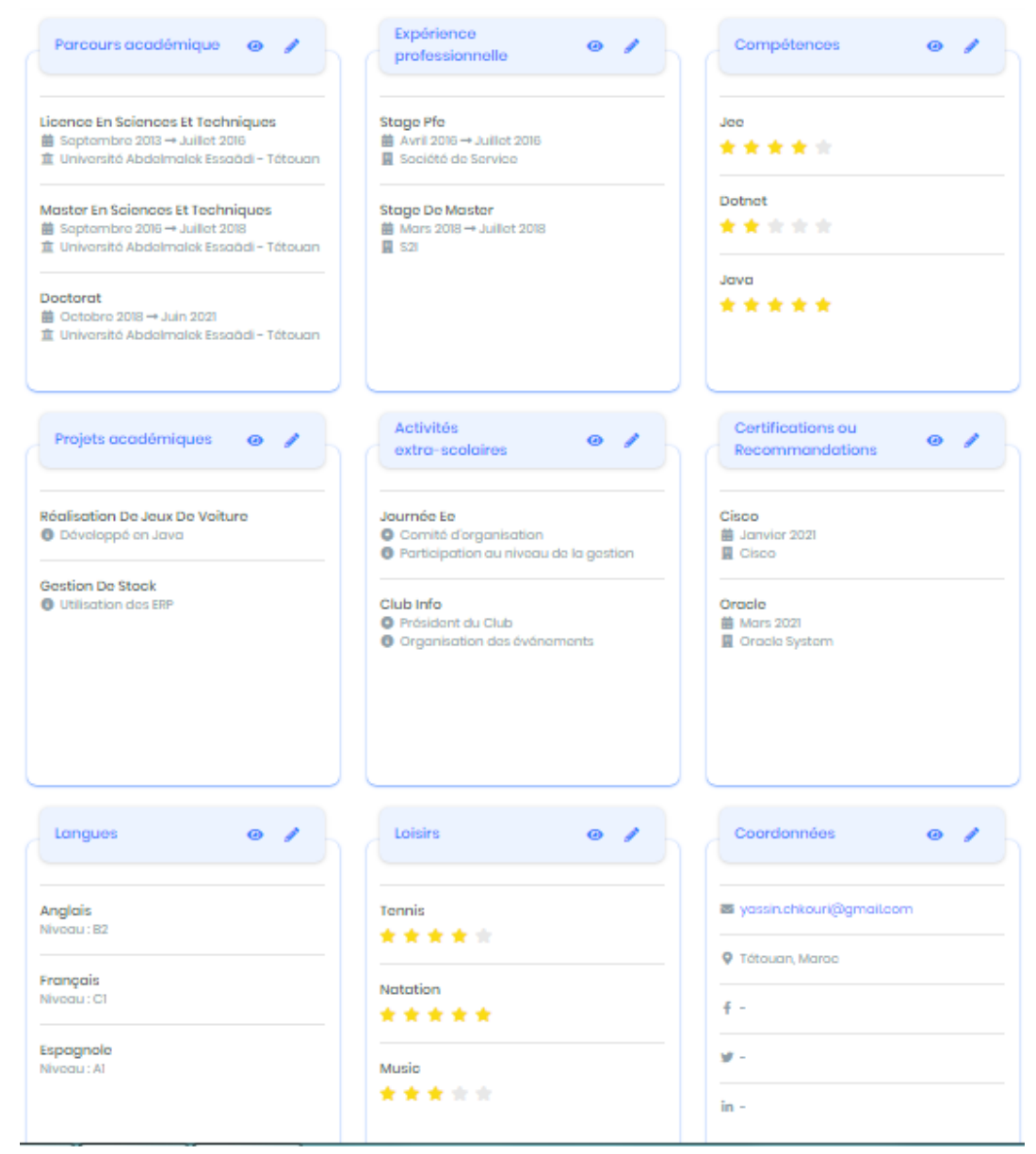

Fig. 3. The sections of the e-portfolio 
The user with simple click can view, add or update the list of academic background as shown in Figure 4.

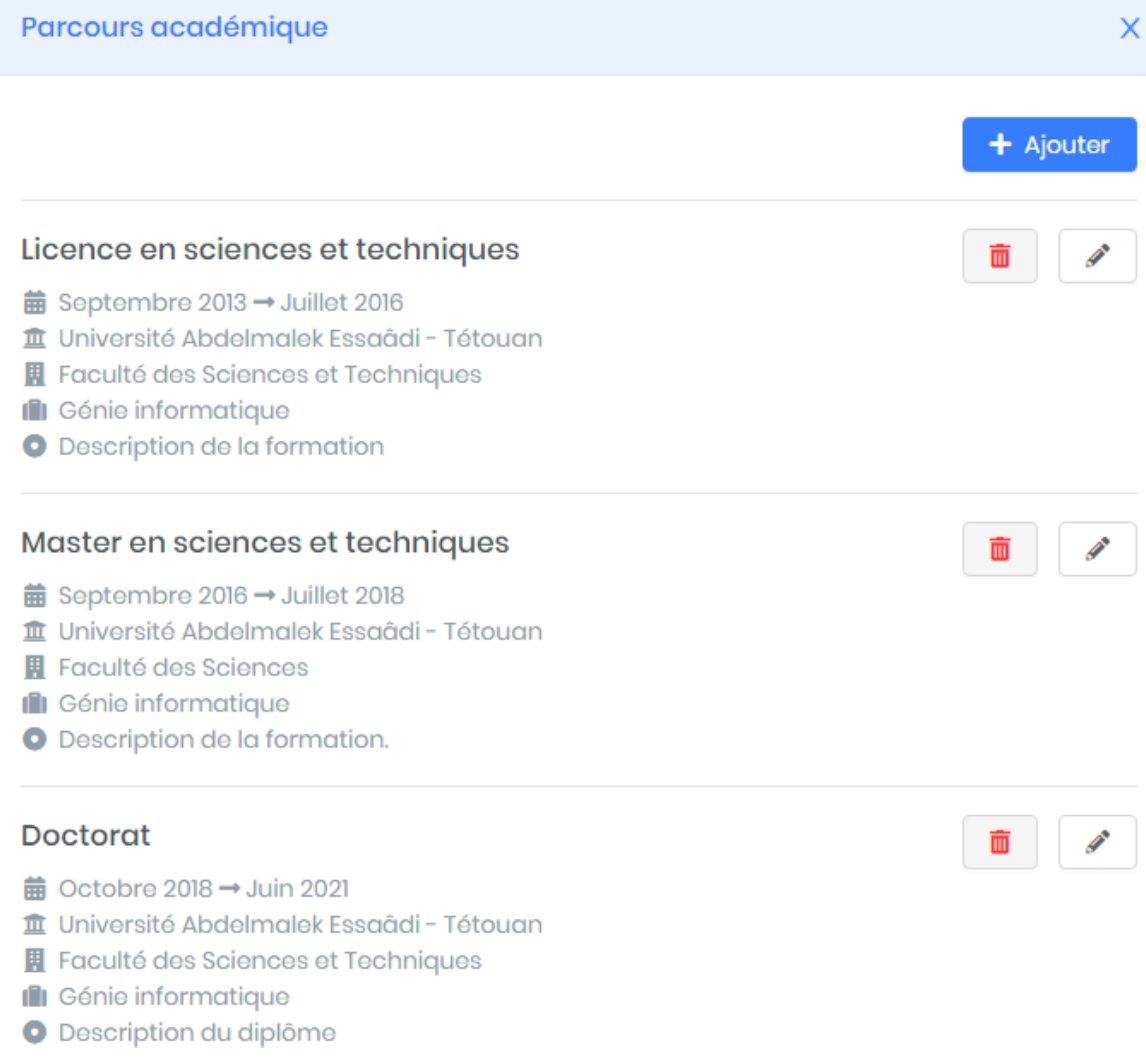

面

Fig. 4. Academic background

Also, he can view, add or update the list of professional experiences as shown in Figure 5.

The student will have the choice of uploading their CV in PDF format or viewing it in Web format. The web link and the CV can then be shared with companies to apply for a position.

The e-portfolio platform is innovative and original in the Moroccan national context. It is oriented towards assisting the professional integration of graduates into the socioeconomic world. It is characterized by:

- The creation of an innovative e-portfolio platform in the Moroccan university context in French and Arabic (to adapt to the target audience);

- Monitoring and capitalization of students' experiences and skills; 
- The promotion of a reflective approach by students on their personal project: personal, professional and training course (initial, continuing and throughout life);

- The development of the culture of digital identity by the student;

- The implementation of new architecture and software engineering techniques and technologies in the e-portfolio platform.

The electronic version of the portfolio has additional potential and possibilities: flexibility, ease updating of the contents and structure of the portfolio, the addition of multimedia documents, accessibility and integration into a large network.

\begin{tabular}{|c|c|c|}
\hline \multirow[t]{2}{*}{ Expérience professionnelle } & \multicolumn{2}{|c|}{$>$} \\
\hline & + & uter \\
\hline Stage Pfe & 面 & $\Rightarrow$ \\
\hline 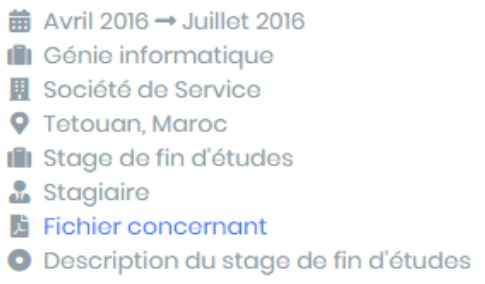 & & \\
\hline Stage De Master & 画 & $\Rightarrow$ \\
\hline $\begin{array}{l}\text { Mars } 2018 \rightarrow \text { Juillet } 2018 \\
\text { 1ill Génie informatique } \\
\text { 田 S2l } \\
\text { 9 Tanger, Maroc } \\
\text { 1ill Stage d'observation } \\
\text { \& Stagiaire } \\
\text { : Fichier concernant } \\
\text { - Description }\end{array}$ & & \\
\hline
\end{tabular}

Fig. 5. Description of the professional experiences

\section{Conclusions}

We establish an online digital environment based on the e-portfolio approach allowing students to develop their digital visibility by capitalizing on the learning outcomes and skills obtained during their studies and after graduation. The e-portfolio platform is innovative and original in the Moroccan university context. It is oriented towards assisting the professional integration of graduates into the socio-economic world by the 
Paper-Purpose and Design of a Digital Environment for the Professional Insertion of Students Based...

development of the culture of digital identity and capitalization of experiences and skills.

\section{$6 \quad$ Future work}

The next steep of this work is to integrate the machine learning to choose the jobs corresponding to the profile of the student. The recommendation system will be able to provide the jobs for applicants already registered on the e-portfolio platform ordered in a dynamic score that indicates the degree of relevance between the candidate profile and job listings.

\section{$7 \quad$ References}

[1] Daunert, Anna Liza and Price, Linda (2014). "E-portfolio: a practical tool for self-directed, reflective, and collaborative professional learning". Discourses on Professional Learning: On the Boundary Between Learning and Working. Vol. 2, P. 231-251. ISBN 978-94-0077011-9. https://doi.org/10.1007/978-94-007-7012-6 13

[2] M. Bhattacharya and M. Hartnett, "E-portfolio assessment in higher education" 2007 37th Annual Frontiers In Education Conference - Global Engineering: Knowledge Without Borders, Opportunities Without Passports, 2007, pp. 19-24. https://doi.org/10.1109/FIE.2007. $\underline{4418182}$

[3] San Jose, David. (2017). "Evaluating, Comparing, and Best Practice in Electronic Portfolio System Use". Journal of Educational Technology Systems. Vol. 45. P. 476-498. https://doi. org/10.1177/0047239516672049

[4] Syzdykova, Zhibek \& Koblandin, Kalybek \& Mikhaylova, Natalia \& Akinina, Olga. (2021). Assessment of E-Portfolio in Higher Education. International Journal of Emerging Technologies in Learning (iJET). Vol. 16. P. 120. https://doi.org/10.3991/ijet.v16i02.18819

[5] R. Mobarhan and A. A. Rahman, "A conceptual model for e-Portfolio continuous use among students integrating Uses and Gratification theory and Information system continuance model," 2014 IEEE Conference on e-Learning, e-Management and e-Services (IC3e), 2014, P. 12-17. https://doi.org/10.1109/IC3e.2014.7081234

[6] R. Zaini, E. Quqandi and W. Guezguez, "A Multi-criteria Decision Making Model for Software Selection to Build E-Portfolio," 2015 Fifth International Conference on e-Learning (econf), 2015, P. 131-134. https://doi.org/10.1109/ECONF.2015.89

[7] M. Sanchez, K. Kephart, K. Jones and M. des Jardins, "A Methodology to Analyze SelfReflection in E-Portfolios," 2020 IEEE Frontiers in Education Conference (FIE), 2020, P. 1-5. https://doi.org/10.1109/fie44824.2020.9274281

[8] P. Rahayu, D. I. Sensuse, B. Purwandari, I. Budi and N. Zulkarnaim, "Review on e-Portofolio definition, model, type and system," 2016 International Conference on Information Technology Systems and Innovation (ICITSI), 2016, P. 1-7. https://doi.org/10.1109/icitsi .2016 .7858204

[9] Stefani, Lorraine \& Mason, Robin \& Pegler, Chris. (2007). The Educational Potential of ePortfolios: Supporting Personal Development and Reflective Learning. ISBN 9780415 412148. https://doi.org/10.4324/9780203961292 
[10] Gerbic, Philippa \& Lewis, Lyn \& Northover, Mark. (2009). Student perspectives of eportfolios: A longitudinal study of growth and development. Proceedings ascilite Auckland 2009: Concise paper: Gerbic, Lewis and Northover.

[11] Beutelspacher, Lisa. "Evaluation des E-Portfolio-Systems Mahara" Information - Wissenschaft \& Praxis, Vol. 63, No. 4, 2012, P. 227-232. https://doi.org/10.1515/iwp-2012-0051

[12] Chau, Juliana \& Cheng, Gary. (2010). Towards understanding the potential of e-portfolios for independent learning: A qualitative study. Australasian Journal of Educational Technology. Vol. 26. P. 932-950. https://doi.org/10.14742/ajet.1026

[13] Kumar, Vikas. (2016). Creating Collaborative and Convenient Learning Environment Using Cloud-Based Moodle LMS: An Instructor and Administrator Perspective. International Journal of Web-Based Learning and Teaching Technologies. Vol. 11. P. 35-50. https://doi. org/10.4018/IJWLTT.2016010103

[14] Duquenoy, Eric \& Dooremont, Frédéric. (2017). Getting Started with Karuta. Conference: Apereo 2017 At: Philadelphia, USA.

[15] V. Ramasamy, J. Kiper, H. Ojha and U. Desai, "Analyzing Link Dynamics in Student Collaboration Networks using Canvas-A Student-Centered Learning Perspective," in 2019 IEEE Frontiers in Education Conference (FIE), Covington, KY, USA, 2019. P. 1-9. https://doi.org/10.1109/FIE43999.2019.9028629

[16] Burrack, Frederick \& Thompson, Dorothy. (2021). Canvas (LMS) as a means for effective student learning assessment across an institution of higher education. Journal of Assessment in Higher Education. Vol. 2. P. 1-19. https://doi.org/10.32473/jahe.v2i1.125129

[17] Clayton, John. (2009). Presenting content to students using the functionalities of Exabis: A portfolio block in Moodle. Conference: Sloan-C International Symposium on Emerging Technology Applications for Online Learning 2009.

[18] I. Balaban and G. Bubas, "Educational potentials of ePortfolio systems: Student evaluations of Mahara and Elgg," Proceedings of the ITI 2010, 32nd International Conference on Information Technology Interfaces, 2010, P. 329-336.

[19] Livre blanc «La démarche ePortfolio dans l'enseignement supérieur français». Ministère de l'enseignement supérieur et de la recherche - service de la stratégie de l'enseignement supérieur et de l'insertion professionnelle (DGESIP), 2013.

[20] Heutte, Jean \& Jézégou, Annie. (2012). La démarche ePortfolio dans l'enseignement supérieur français: Retours d'expériences et proposition de clarification. 24ème colloque de l'Association pour le développement des méthodes d'évaluation en éducation (ADMEE 2012) At: Luxembourg.

[21] Jafari, A. \& Kaufman, C. Handbook of Research on E- Portfolios, Idea Group Publishing. IGI Global, 2006. ISBN: 9781591408918.

[22] M. Baidada, S. Carolan, N. El Mawas, F. Poirier, M. Rabah. Portfolio dans la formation tout au long de la vie. Actes de l'atelier L'apprenant, acteur principal de son parcours tout au long de sa vie. Atelier des ORPHEE-RDV. 2017.

\section{Authors}

Hanae Mgarbi is PHD student at the research laboratory of the Information System and Software Engineering (SIGL) at National School of Applied Sciences, and the computer engineering at the Abdelmalek Essaadi University, Tetouan, Morocco.

Mohamed Yassin Chkouri is the leader and founder of the accredited research laboratory of the Information System and Software Engineering (SIGL) and the Head of 
Department of Computer Engineering since 10/2016 at National School of Applied Sciences, Abdelmalek Essaadi University, Tetouan, Morocco (Email: mychkouri@uae .ac.ma).

Abderrahim Tahiri received his engineering degree and Ph.D. in Computer Science from the University of Abdelmalek Essaädi-Morocco. Since 2010, he has been an Associate Professor in the Department of Informatics at the National School of Applied Sciences of Tetouan, School of Engineering at Abdelmalek Essaädi University. He has been a member of the school Council and was appointed Coordinator of the Informatics branch then head of department (Email: t.abderrahim@uae.ac.ma).

Article submitted 2021-09-03. Resubmitted 2021-10-12. Final acceptance 2021-10-15. Final version published as submitted by the authors. 